\title{
¿Cómo hacer escuelas democráticas?
}

J osep Ma Puig Rovira

UniversitatdeBarcelona

Resumen

Para responder a la pregunta que titula el presente artículo, "¿Cómo hacer escuelas democráticas?", se ha llevado a cabo un rodeo que nos ha conducido, en primer lugar, a definir qué se entiende por escuela democrática, a ver cuáles son sus principalesli mitacio nesy er ro res, yquépo si bilidadeslequedan to davía como instrumento educativo de transformación social. La escuela democrática se ha caracterizado como un espacio de participación que, sin embargo, muy a menudo ha producido desigualdad y discriminación. Pese a todo, las escuelas democráticas deben ser espacios de controversia y lucha en favor de la democracia.

En segundo lugar, el artículorealiza un rápido recorrido por al gunos de las principales tendencias y momentos de desarrollo de las escuelas democráticas.

Luego, en su tramo final, el escrito propone un conjunto de di namismos pedagógicos que han de ayudar a construir escuelas entendidas como comunidades democráticas. Dinamismos como los en cu en tros, laformación depequeñosgru pos, la definición de espacios de diálogo y participación, y el diseño de prácticas de valor.

Finalmente, el tex to con clu yecon la presen ta ción de una delas prácticas esenciales de las escuelas democráticas: las asambleas de clase. Además de presentar qué son las asambleas y cómo deben funcionar, se analiza su papel en tanto que instrumento de educación en valores y preparación para la ciudadanía.

Palabras clave

Escuela democrática - Participación - Diálogo - Asamblea de Clase.

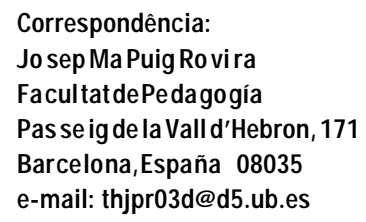




\section{How to make democratic schools?}

J osep Ma Puig Rovira

UniversitatdeBarcelona

\begin{abstract}
In order to answer the question in the title of this article, a journey was carried out, what made us, in first place, to define what is understood by democratic school, what are its main limitations and errors, and what are its possibilities as an instrument of social changes. The democratic school has distinguished itself as a locus of participation that has, however, frequently produced inequality and discrimination. In spite of that, democratic schools must be spaces for controversy and fight for democracy.

In second place, the ar ti clema kes a bri ef revi ew of some of the main trends and moments of the development of democratic schools.

Then, in its final part, the text proposes a set of pedagogical dynamics that should help to build schools as democratic communities. Dynamics such as meetings, the use of small groups, the definition of spaces for dialogue and participation, and the design of value practices.

Finally, the author concludes describing one of the essential practices of democratic schools: classroom assembly. A part from the description of these assemblies and how they are supposed to work, their role is also analyzed both as an instrument of values education and as a preparation for citizenship.
\end{abstract}

Keywords

Democratic school - Participation - Dialogue - Classroom Assembly.
Correspondence:

J o sep Ma Puig Ro vi ra

FacultatdePedagogía

Pas se ig de la Vall d'Hebron, 171

Barcelona, Espa ña 08035

e-mail: thjpr03d@d5.ub.es 
Para intentar responder a la pregunta que titula este escrito, ¿Cómo hacer escuelas democráticas?, vamos a dar un rodeo que nos llevará a tratar las siguientes cuestiones: primero, esbozar una definición de lo qué se entiende por escuela democrática y constatar cuáleshan sido susprin ci palesli mi taci o nes; segundo, trazar un rápido panorama de algunos momentos importantes en el desarrollo de las escuelas democráticas; tercero, definir los dinamismos pedagógicos fundamentales que intervienen en la construcción de escuelas democráticas; y, cuarto, presentar las asambleas de clase como una de las prác ti casesen ci a lesen la construcción de escuelas democráticas.

\section{La escuela como comunidad democrática}

Con la expresión "escuela como comunidad democrática" queremosreferirnos al espíritu que ha animado la política educativa al menos desde la llustración. Por una parte, la igualdad, la libertad y la autonomía en tanto que condiciones de la participación social y, por la otra, la transmisión de un tipo de conocimientos que impulsen la crítica, la emancipación personal y el progreso colectivo. Ambas líneas han sido y son todavía el horizonte de valor de la escuela democrática. Como resulta obvio, el ám bi to de temasafec ta do por una po lítica de implantación deescuelasdemo cráticas es muy amplio y no es posible abordarlo completamente. Por lo tanto, y aunque los ideales democráticos se han expresado de múltiples maneras, aquí vamos a destacar tan sólo los es fuerzos por garantizar la autonomía y la participación de todos los miembros de la comunidad educativa: los esfuerzos por hacer una escuela abierta a la libre participación de todos sus miembros.

Democracia y educación

La tendencia educativa que hemos denominado "escuela como comunidad democráti- ca" agrupa aquellas ideas y realizaciones preocupadas por crear formas escolares que preparen para la convivencia democrática y que permitan el ejercicio real de la autonomía y la participación. Tales experiencias reflejan y a la vez impulsan la democracia. La "escuela como comunidad democrática" es la expresión de una voluntadcul tu ral ypolítica propia de los regímenes democráticos. Estamos ante un reflejo escolardela demo craciapolítica. La escuela debe ser un espacio social regido por los criterios de participación propios de los sistemas democráticos.

Los vínculos entre educación y democra cia sehan en ten di do tam bién en una di rección inversa. Los ideales democráticos inspiran sin duda prácticas educativas democráticas, pero se espera que estas realizaciones educativas contribuyan decisivamente a impulsar proyectos de transformación social. La escuela ha de preparar a todos sus integrantes, y de modo especial a sus alumnos y alumnas, para que sean capaces de asumir en el futuro las ideas y hábitos democráticos. Aquí la escuela es un instrumento para reproducir, consolidar y optimizar la democracia.

Pero la escuela reflejará e impulsará la democracia en la medida que en su interior se lleven a cabo prácticas democráticas. Es decir, si por encima de todo es un espacio de autonomía y participación. Si enseña a participar participando, o si enseña autonomía dejando decidir reflexivamente. Por lo tanto, y en primer lugar, una escuela democrática será aquella que sepa organizarse de modo que estimule la participación de todos los implicados: que reconozca como interlocutores válidos a todos sus miembros. Todos son tenidos en cuenta en tanto que participantesen un diálogo que tomará sin lugar a dudas formas distintas, pero que no excluirá a ninguno de los miembros de la comunidad. En segundo lugar, las experiencias escolares democráticas suponen la participación de los miembros de la comunidad en las diversas 
tareas que allí se llevan a cabo. La participación no es un principio formal, sino el ejercicio de una tarea en alguno de los ámbitos de acción de la escuela. Cabe destacar al menos los siguientes ámbitos: la participación en la gestión del centro, la participación en la elaboración y aplicación de proyectos, planes y programaciones docentes, y la participación en el trabajo escolar y en la regulación de la convivencia en el grupo-clase y en el conjunto del centro. En tercer lugar, las experiencias escolares democráticas suponen que la comunidad escolar es capaz de poner en marcha instancias de participación adaptadas a cada uno de los ámbitos de acción de un centro. Tienen ya una cierta tradición los consejos escolares, los claustros, las asambleas de clase o las actividades de tutoría, aunque no deberíamos considerar estas vías como las únicas oportunidades de participación. En cuarto y último lugar, la participación de la comunidad escolar es un fin en sí misma, ya que expresa valores democráticos, y a la vez es el mejor medio para alcanzar otros fines propios de la tarea educativa. Las experiencias escolares de participación y autonomía son un medio, un medio entre otros, para lograr el pleno desarrollo de la personalidad en el interior de una sociedad justa, libre y solidaria.

Ambigüedades y fracasos de la escuela democrática

Hoy sería de una ingenuidad y un desconocimiento enormes dejar aquí la caracterización de la escuela democrática. Sin duda se han proclamado con insistencia los criteriosorganizativos y las finalidades que acabamos de exponer, pero en la actualidad sabemos que la escuela tiene una cara cada vez menos oculta que niega justo aquello que públicamenteafirma. Podemos decir a partir de las aportaciones de múltiples autores que la escuela democrática es una realidad profundamente contradictoria. Respecto a lo que aquí nos ocupa, la escuela defiende la participación y la autono- mía, y a la vez se comporta de modo autoritario y heterónomo (Santos Guerra, 1995; Fernández Enguita, 1992). Pero veamos aunque sea muy rápidamente algunas de las prin cipales contradicciones que se viven en la escuela.

La escuela como institución igualitaria que, sin embargo, reproduce la desigualdad social. La so cio olo gíahamostra do comola escuela pese a su discurso y a sus prácticas igualitaristas, en realidad está facilitando el éxito de tan sólo una parte de la población.

La escuela como institución respetuosa con las diferencias y garan tedela tolerancia que, sin embargo, inocula actitudes discriminatorias. La escuela defiende la libre expresión de las diferencias en un marco de valores como la libertad, la igualdad y la tolerancia, pero a menudotransmite de modo im plícito u oculto actitudes sexistas, racistas, xenófobas y competitivas.

La escuela como institución que proclama la necesidad de un aprendizaje crítico y creativo pero que, sin embargo, usa medios verbales y memorísticos. Junto a la defensa de criterios de aprendizaje como la actividad, la crítica o la creatividad, la escuela ha acaparado también infinidad de denuncias sobre el carácter jerárquico, pasivo, repetitivo y alejado de la realidad del apren dizajequepro picia.

La escuela como institución democrática que, sin embargo, está atravesada por hábitos autoritarios que limitan la participación y la autonomía. Como ya hemos apuntado, la escuela ha querido ser una institución democrática, aunque el autoritarismo haya sido un modo corriente de operar.

La escuela como espacio de trabajo en favor de la democracia

Hemos visto en qué medida resulta imposible quedarsecon unacaracterización idealizada de la escuela: la escuela no es una comunidad democrática perfecta. Pero tampoco es correcto verla únicamente como un 
instru men to derepro ducción de desi gual da des, de creación de prejuicios y de convivencia autoritaria: la escuela no es una realidad de naturaleza perversa. La escuela es un teatro de luchas y un espacio de compromiso en favor de la democracia. El futuro de la escuela no está escrito en ninguna esencia intrínseca ni en nin guna ley social inexorable. El futuro dependerá del trabajo que sobre ella lleven a cabo los implicados, desde la administración, a los alumnos y alumnas.

La tendencia educativa que aquí estamos considerando, la "escuela como comunidad democrática", no tiene, pues, un carácter monolítico. Ciertamente está animada por valores como la participación y la autonomía, pero la entenderemos mejor si la imaginamos como un espacio de controversias donde se producen avances y retrocesos. Se trata de caracterizar una tendencia de largo recorrido que está sujeta, como toda realidad dialéctica, a oscilaciones y altibajos.

En lo sucesivo vamos a considerar una línea de trabajo que sin ser la única constituye sin duda una realización muy significativa en ese esfuerzo inacabado por construir una escuela democrática: nos referimos a las experiencias de autogobierno escolar.

\section{Experiencias de autogobierno escolar}

Términos como autonomía, autogobierno, participación o autogestión son algunos de los más usados para referirse a la "escuela como comunidad democrática". No obstante, aquí todos estos conceptos se refieren menos a la gestión global de los centros educativos y más a la creación de un clima de aula democrático. Es decir, a la crea ción deuna am bi en tequepermita a los educadores poner en manos del alumnado la organización de todas aquellas cuestiones que sea posible confiarles $y$, en cualquier caso, que permita abrir espacios de diálogo para tratar los temas que afectan al grupo-clase. Las experiencias de autogobierno escolar suponen pues la creación de canales de diálogo y de acción cooperativa.

La escuela autoritaria

Iniciar un breve recorrido por entre las distintas experiencias de autogobierno escolar precisa necesariamente dibujar primero el fondo sobre el cual todas ellas se destacan. Nos referimos a la pedagogía tradicional y, de modo más preciso, a la escuela que defiende valores absolutos y se basa en instrumentos como el autoritarismo y la disciplina represiva. Tal escuela dice tener una imagen muy clara de cómo deben llegar a ser los seres humanos y de cómo imponer esa imagen. En or den a conseguirlo usará el castigo abundante e indiscriminado como medio para reparar cualquier transgresión en que incurran los alumnos. 0 bien, de modo mucho más inteligente y sutil, creará unas condiciones espacio-temporales y de vigilancia para modelar las conductas y las mentes de manera que no haya lugar para la desviación. La consecuencia de este tipo de pedagogía es la creación de un ambiente escolar autoritario que desconoce del todo la libertad y la autonomía de los escolares, y que inocula temor a los adultos, desconfianza entre los alumnos y actitudes competitivas.

La escuela democrática

Las primeras experiencias de democracia escolar se piensan y se llevan a cabo para enfrentarse a las tradiciones autoritarias y para impulsar en la escuela los criterios éticos quesu po nelademo cracia po lítica. El mo vimiento de la Escuela Nueva y los autores implicados en él son los mejores representantes de esta etapa de la democratización escolar (Dewey, 1918; Ferriere, 1928). En todos los casos se parte de una confianza absoluta en la bon dad de la democracia y en la posibilidad de imitar, a veces casi literalmente, su funcionamiento en la escuela. La experiencia de las 
repúblicas escolares, por una parte, 0 de las co operativas y consejos de clase, por otra, fueron las realizaciones más representativas. En cualqui ercaso, se tra ta de apli car el prin ci pio de actividad no sólo a los aprendizajes intelectuales, sino también al aprendizaje de la convivencia y la ciudadanía. Estas experiencias suavizaron enormemente el autoritarismo y la disciplina escolar, abrieron espacios de libertad, participación y autonomía, aunque no pretendieron abolir la autoridad de los adultos, ni un marco suficiente de normas y hábitos escolares.

El ideal democrático bajo sospecha y vías de transformación

Bajo este epígrafe vamos a incluir un conjunto de tendencias pedagógicas muy dispares - la pedagogía anarquista, marxista, psicoanalítica, no directiva, institucional o cooperativa -, que en relación a la cuestión que nos ocupa presentan algunas similitudes importantes.

En primer lugar, estamos ante un intento de radicalizar la participación de los alumnos en la vida de las instituciones educativas. La mayoría de estas experiencias, aunque no todas, conceden una libertad casi absoluta a los alumnos y tienden a diluir por completo la autoridad de los educadores. Las propuestas psicoanalíticas, no-directivas e institucionales cumplen en gran medida este criterio (Neill, 1978; Rogers, 1975; Lobrot, 1976). Por su parte, lo mejor de la pedagogía marxista combina muy originalmente un elevado grado de participación con una notable presión de la colectividad y de los educadores (Makarenko, A. 1983). Asimismo, la Escuela Moderna de Freinet y el Movimiento de Cooperación Educativa que se desarrolló en Italia conceden un elevado papel a sus alumnos, aunque no lleguen nunca a plantear situaciones de libertad absoluta (Freinet, 1972; Lodi, 1973).

En segundo lugar, tal radicalización de la libertad se unió, en muchos casos, a una crítica a la democracia política por encubridora de injusticias y por represiva. Se han diluido las esperanzas en la democracia: la escuela debe preparar algo mejor. Las imágenes de la revolución comunista nutren muchos ideales edu cativos y la pretensión del psicoanálisis de instaurar una sociedad liberada impregnó otras tantas realizaciones educativas. Estamos ante una pedagogía que quiere contribuir a un cambio civilizatorio que vaya más allá de las formas democráticas imperantes.

Finalmente, algunos autores señalan la existencia de dinamismos ocultos que explicarían laslimitaci o nes delareali dad perso nal, social y política. Sobre ellos convendría intervenir para liberar de verdad todas las posibilidades humanas y construir una vida más justa, más feliz y más participativa. La explotación y la lu cha decla ses, la represión del deseo o la burocratización de todos los órdenes de la vida son los obstáculos contra los cuales luchan las pedagogías radicales.

La voluntad de las pedagogías radicales entrelaza las ideas de autogestión, transformación política de la sociedad y liberación de todas las trabas que limitan la libre expresión de los seres humanos.

Ni ideal democrático ni esperanza de transformación: cris is

La mayoría de experiencias radicales ha dejado un rastro en la memoria pedagógica, pero su influencia puede decirse que casi ha desaparecido por com pleto del debateedu cativo actual. Se han señalado varias causas para explicar este hecho. Veamos algunas: el exceso de confianza en la posibilidad y la conveniencia de diluir el poder de los educadores, el exceso de politización en la reflexión ylapráctica pedagógica, la caída de los ideales políticos que habían al entado las experienciasradicales, el pesimismo que a veces han provocado las sociologías críticas al no dejar resquicios para la esperanza, y sobre todo la enorme presión eficacista, tecnológica y neoconservadora que ha impregnado el pensamiento educativo. 
Estos y otros factores han propiciado la crisis de la idea de autogobierno escolar. Puede decirse que hoy no es un tema en la agenda educativa. Las experiencias de autogobierno nunca han sido representativas de lo que ocurría en la mayoría de las escuelas, pero en cambio su discusión había ablandado muchas realidades, obligado sin duda a flexibilizar posiciones $y$, al fin, a mejorar lentamente la realidad escolar. La situación hoy es distinta: casi no hay siquiera debate. Se combina un laissez-faire pretendidamente democrático, un autoritarismo tradicional y ciertos rituales democráticos acompañados de escasas convicciones. De esa mezcla surge el desencanto que en relación a la participación viven tanto los profesores como los alumnos.

Sin embargo, recientemente va tomando protagonismo una renovada preocupación por la participación de los alumnos y alumnas. A menudo es una preocupación que se ha vuelto a plantear de la mano de la reflexión sobre la educación moral. La tesis es muy clara: además de un tratamiento sistemático y transversal de temas que implican valores, la mejor educación moral es aquella que permite reflexionar y actuar sobre la misma convivencia del grupo-clase. Por lo tanto, se trata de recuperar desde otra perspectiva la vieja idea de la autonomía y del autogobierno de los escolares. Reforzando esta posición, lentamente nos van llegan do lostrabajos deL. Kohl berg so brela escuela como comunidad justa (Power; Higgins; Kohlberg, 1989). Trabajos que en muy buena medida defienden la posición clásica que entiende la participación como medio para construir una convivencia colectiva justa.

\section{Dinamismos de una escuela democrática}

Hasta aquí nos hemos aproximado a la idea de comunidad democrática y hemos recorrido algunos momentos de su historia, ahora nos dirigiremos en otra dirección: vamos a ver qué tipo de dinamismos humanos deben institucionalizarse para construir una escuela demo crática. La tesis que vamos a defender es muy sencilla: una escuela democrática se construye dando forma institucional a ciertos dinamismos básicos de relación humana. A saber, la democracia en la escuela depende de que se puedan cristalizar en prácticas pedagógicas la relación afectiva, la deliberación y la cooperación.

Modalidades de la relación social

Si partimos del supuesto de que la democracia escolar no depende de la mera voluntad individual, ni tampoco de la existencia previa de una comunidad que ya da por supuesto lo que se trata de crear, sólo nos queda intentar determinar con mayor precisión el primer eslabón de la democratización de las escuelas. En ese primer eslabón no encontramos unidades aisladas - individuos - , ni totalidades acabadas - comunidades - , sino procesos de relación entre sujetos. En el origen tanto de la individualidad como de la comunidad lo que hay son encuentros entre sujetos: relación interpersonal. En esta dirección empezaron a caminar con cierta seguridad G.H. M ead (1982) y L. S. Vi gotsky (1979) y en la actualidad, entre otros, ha seguido avanzando J. Habermas (1990).

Una vez establecido que en la relación tenemos el principio explicativo de la democraciaescolar, debemospreci sarlasmo dalidades de la relación entre sujetos. Como veremos, setrata demo dali da dessi em prepresentes en la acción humana, aunque se manifiesten de diversa manera y con distintos pesos en cada uno de los espacios o instituciones sociales. Además estamos ante modalidades que reconoceremos en su forma más abstracta y general, no en sus manifestaciones contextuales y concretas. Y, por último, se trata de caras de la relación social que sólo con voluntad expositiva podemos separar, ya que en la realidad presentan una textura 
compleja y entremezclada. Hechas estas advertencias, decir que consideramos que la relación humana se expresa a través de vínculos afectivos, comunicativos y cooperativos. Es decir, la democracia depende del entramado que construyen las relaciones afectivas entre sujetos, las múltiples formas de deliberación social y las prácticas de cooperación.

Las relaciones interpersonales basadas en el afecto son el primer nivel de la democracia esco lar. El afec to y la amistad no pu eden ga ran tizar completamente la democracia, pero constituyen una de sus fuerzas originarias y sin duda imprescindibles. Adquieren esa función porque son la primera experiencia de la moralidad; es decir, el im pul so mo ral nacedela rela ción afecti va con los demás: las vivencias del otro nos conmueven y nos aproximan a él con tal fuerza que llegan a producir una intensa motivación altruista y un profundo lazo social. La simpatía que brota del contacto interpersonal, pese a quedar limitada a sujetos próximos con los que podemos relacionarnos, es una experiencia imprescindible para la democracia y la participación escolar (Hume, 1988; Aristóteles, 1982).

La comunicación es otra de las modalidades de la relación y una fuente esencial de democracia. La acción comunicativa es una fuerza que im pul sa lacom pren sión mu tuay, en consecuencia, produce efectos de integración social. Cuando los participantes en una conversación se reconocen mutuamente y se comprometen en un intercambio de argumentos con ánimo de alcanzar una mejor comprensión y a ser posible un acuerdo libre, se produce entre ellos un incremento de los lazos sociales y de la cohesión. Por otra parte, la acción comunicativa permite establecer una propuesta normativa sobre lo que puede ser la ciudadanía: la participación en un diálogo generalizado que aborde los problemas de la colectividad y que ayude a formar la opinión y la voluntad de los ciudadanos. Por lo tanto, en las prácticas deliberativas tenemos una de las más potentes fuerzas democráticas (Habermas, 1987, 1998, 1996).
Las prácticas cooperativas son la última modalidad de la relación: otro de los dinamismos de la democracia escolar. Por prácticas de cooperación vamos a entender las acciones concertadas de varios sujetosque se encaminan a alcanzar una finalidad deseada. Las prácticas exigen acciones individuales que se coordinan creando cualidades y efectos no explicables por ninguna de las aportaciones parciales. La contribución de cada sujeto es necesaria para alcanzar el resultado final, pero lo que resulta de la participación de todos los implicados tiene unos efectos y unas cualidades que sobrepasan las contribuciones individuales. En las prácticas emergen cualidades nuevas, pero sobre todo se crean ataduras sociales por coordinación de acciones y se comparten valores que facilitanlademocracia (Dworkin, 1996).

En síntesis, la democracia escolar no se logra profundizando en el individualismo con la esperanza de que las acciones se coordinen a partir de la búsqueda de beneficios individuales, ni se logra apelando a la existencia ontológica de una comunidad ya cohesionada. Sino que la escuela como comunidad democrática y participativa resultadela rela ción interpersonal que se concreta en el afecto, la comunicación y la cooperación. Por tanto, y a modo de consecuencia, consideramos que cabe orientar las políticas institucionales hacia la reconstrucción de espacios y procesos sociales propicios para la calidez afectiva, para el diálogo y para la cooperación. Espacios y políticas que en unos casos, como el nuestro, deben adaptarse a la naturaleza de las institucioneseducativas, pero que en otros casos deberán tener en cuenta las particularidades de las demás instituciones sociales.

Comunidad y democracia en la escuela

En este apartado vamos a esbozar una traducción que nos permita aplicar las modalidades de la relación - afecto, diálogo y cooperación - a las circunstancias de la institu- 
ción escolar. Se pretende establecer una política educativa y unas formas de intervención escolar que dibujen las líneas que definen una comunidad escolar democrática.

Cuando proponemos la creación de comunidades escolares democráticas no estamos pensando en escuelas uniformes, sino en escuelas plurales en las que muy probablemente van a convivirprofeso res y alumnos de distintaprocedencia étnica, con diferentes raíces culturales y con valores también diferentes. Una comunidad democrática no es una comunidad homogénea, sino una comunidad diversa y a la vez integrada gracias a los efectos que produce la relación afecti va, el diálo go yla co o peración.

Una comunidad democrática debe organizarse de manera que sus miembros puedan contar con frecuentes ocasiones, previstas e imprevistas, para mantener encuentros cara-a-cara y relaciones interpersonales continuadas. Los encuentros y las relaciones frecuen teshan depermitirqueen trepro feso res y alumnos se creen lazos de afecto. Lazos que son una condición imprescindible para llevar a cabo con éxito cualquier proceso de educación moral. Cu an do hay vo lun tad edu ca ti va demantener abiertos los canales de relación con los alumnos, y si se cuenta con momentos y lugares adecuados para establecer tal relación, se reúnen las condiciones mínimas para conducir procesosdeaco gi da delosalum nos, dereconocimiento y de aceptación, que al fin desemboquen en la creación de nudos de confianza y de afecto. Sin una relación interpersonal de estas características no resulta imaginable establecer una comunidad, ni tampoco resulta posiblegenerar sentimientos de obligación, vergüenza, culpa y orgullo. Sentimientos que, en su justa medida, constituyen fuerzas esenciales para la integración social y la ciudadanía.

Una comunidad democrática debe organizarse de manera que impulse la asociación entre iguales. La relación de amistad entre los alumnos y, sobre todo, la formación de pequeños grupos constituye un componente funda- mental de una comunidad educativa. Los alumnos no pueden quedar convertidos en cuadros vivos o en masas sin forma, ni tampoco debe quedar relegada su capacidad de aso ciación en grupos al ámbito de lo subterráneo de la institución. Los grupos de iguales son una de las fuerzas educativas más importantes con que cuentan los educadores. La emulación, la responsabilidad, la cooperación, la solidaridad y otras virtudes se aprenden y se aplican en primerlu garen esa instan ciaso cial primaria que son los pequeños grupos de iguales. En consecuencia, es tarea de los educadores impulsar su creación, mantener su vida, propiciar su desarrollo y valorar conjuntamente con los alumnos sus efectos y resultados.

Una comunidad democrática debe ser un espacio de diálogo. Para lograrlo conviene establecer mecanismos institucionales destinados a que todos sus miembros deliberen, de manera sistemática u ocasional, sobre todas aquellas cuestiones que les afectan. Se trata de que adultos y jó venespu edan debatirso bre todo lo que les ocurre y sobre todo lo que ocurre en la sociedad. Este intercambio constante, que puede concretarse en distintos espacios y tiempos escolares, ha de facilitar la creación o recreación del sentido social y la identidad personal. Compartir puntos de vista sobre temas vitales y significativos es uno de los mejores medios de integración social y de ciudadanía.

Una comunidad democrática debe dar posibilidades de participación real a todos sus miembros. Con ello se pretende que el hecho de "tomar parte" o "intervenir" abra las puertas de la implicación personal en la institución de manera que todos puedan sentirse parte de ella y estar suficientemente motivados como para mantener vivos los lazos normativos. La participación a través del diálogo incrementa la comprensión mutua, la elaboración de formas de convivencia y el compromiso por realizar todo aquello que se ha 
acordado. Finalmente, los procesos de participación en la institución escolar deberían referir seal tra ba jo, a la con vi ven cia y a lasac ti vi dades de animación. Sin posibilidades de participación la integración y la ciudadanía no son posibles de ningún modo.

Una comunidad democrática debe instituir prácticas de valor que faciliten la cooperación entre sus miembros y la experiencia de valores encarnados en formas de comportamiento. Se trata que la escuela establezca surcos de valor por donde circulen las actividadestanto de alumnos como de profesores. Por tanto, las prácticas de valor son realizaciones escolares que coordinan la acción de diversos miembros de la co munidad escolaren labúsquedade unos fines que se consideran valiosos y a través de unos procesos que también expresan valores. Las principales prácticas de valor en una escuela se refieren al aprendizaje, a la convivencia y a la animación. Algunas de las prácticas de valor más características del ámbito de los aprendizajes son los métodos de aprendizaje cooperativo, la ayuda mutua o la realización de proyectos. En el ámbito de la convivencia pueden destacar los cargos, las asambleas escolares 0 , entre otras, las tareas de mediación en conflictos. Finalmente, en el ámbito de la animación resulta esencial referirse a las fiestas y conmemoraciones, la organización de actividades de tiempo libre y deporte, la confección de revistas o la realización de campañas cívicas. Las prácticas de valor son una parte esencial del contenido de una comunidad democrática que quiera ser fuente de experiencias que faciliten la integración social y la ciudadanía.

Aunque hemos recorrido algunas de las notas más características de una comunidad democrática, merece la pena citar al menos algunas cuestiones finales que no deberían pasar desapercibidas: en primer lugar, el impulso que debe experimentar la reflexividad personal e institucional en todas las facetas en que puede expresase; en segundo lugar, la necesaria ela- boración de un horizonte de valores que exprese un mínimo común dentro de la diversidad de perspecti vasquecon vi ven en un centro edu cativo; en tercerlu gar, la cristalización de rituales, rutinas y normas que hagan previsible y óptima la convivencia cotidiana; en cuarto lugar, la abertura de la institución escolar al entorno próximo y lejano; y, en quinto lugar, la transmisión a todos sus miembros del convencimiento que merece la pena luchar por recu perarla esperanza, in cluso cuando la razón parecenegarcual qui erposibilidad.

Una comunidaddemocrática cuyo objetivo sea impulsar la integración so cial yla ciudadanía deberá concretar estas propuestas, y probablemente otras, en función de sus circunstacias particulares, lo cual no es sino otra de las cualidades que debe expresar toda comunidad democrática: la voluntad de contextualizar y singularizar la manifestación de las relaciones de afecto, diálogo y cooperación.

Cómo hacer cosas con palabras: las asambleas de clase

Este último apartado quiere ser un alegato en favor de las asambleas de clase. Esta práctica educativa no es una pérdida de tiempo, ni una injustificable concesión a los alumnos, ni tampoco es un rasgo residual de las pedagogías radicales de finales de los sesenta. Las asambleas de clase son un elemento esencial en una escuela democrática y un instrumento insustituible de la educación en valo res.

¿Qué es una asamblea?

Solemos decir que las asambleas son el momento institucional del diálogo: un espacio que la escueladestina de maneraexclusiva a fomentar la participación por medio de la palabra. Du ran telasasambleasla cla sesereune para reflexionar sobre sí misma, parato mar conciencia de sí misma y para transformarse en todo aquello que sus miembros consideren 
oportuno. Una asamblea es un momento escolar organizado para que el grupo-clase y sus educadores puedan hablar de todo aquello que les parezca pertinente en orden a optimizar el trabajo, la convivencia y la animación. Para alcanzar estos objetivos, la organización de las asambleas requiere:

- Destinar una pequeña parte del tiempo semanal a este tipo de reunión, de manera que todos consideren la asamblea como una actividad habitual de la clase que pueden usar para alcanzar diversas finalidades.

- Disponer el espacio de la clase de una manera a veces distin taa lahabitual parafa vo recerel diálogo y para fortalecer con estesimbolismo la actitud de cooperación entre todos sus miembros.

- Interrum pir el trabajo ha bi tual del aula ymodificar en cierto modo los roles de alumnos y profesores de manera que su participación sea más igualitaria, aunque no idéntica ni con igual responsabilidad.

- Emplear el tiempo asignado a la asamblea para hablar juntos de todo aquello que le ocurre al grupo-clase, o de todo aquello que cualquiera de sus miembros considera importante y merecedor de la atención del resto de compañeros.

- Dialogar con ánimo de entenderse, de organizar el trabajo y de solucionar los conflictos de relación que puedan plantearse.

- Dialogar pues con la voluntad de cambiar lo necesario para que la vida del grupo-clase sea más óptima y hacerlo con la voluntad de comprometerse personalmente en talescambios.

- Las asambleas, en tanto que reunión para hablar de aquellas cuestiones relevan tespara el grupo-clase, pueden prolongarse e incrementar su efectividad si se completan con otras fórmulas de reunión y debate de la comunidad escolar. Mecanismos como el consejo de delegados, los pequeños grupos de tutorías o la comisión de mediación pueden ejercer esa función.
- Finalmente, el modo de realizar las asambleas de clase depende de la edad del alum nado. Es evidente que las asambleas no pueden llevarse a cabo del mismo modo en la educación infantil o en la educación secundaria. Su necesidad y utilidad pueden ser parecidas, pero la manera de concretarlas variará en función de la especificidad propia de cada edad.

Participar en el trabajo escolar, la convivencia y la animación

La participación que se lleva a cabo en las asambleas se concreta en el trabajo, la convivencia y la animación. Es decir, se trata de tomar parte activa en la regulación de estos tres ámbitos de la vida escolar.

Resulta claro que la escuela es una institución cen trada en el apren di zaje. Portan to, uno de los ámbitos naturales de participación será precisamente el del trabajo escolar. Sería un contrasentido reclamar una escuela democrática y no aplicar sus principios a su principal objetivo. La escuela debería buscar fórmulas para facilitar que sus alumnos pudieran organizarse y responsabilizarse de espacios paulatinamente más amplios de su proceso de aprendizaje. Muchos aspectos de este proceso están en ma nos delosedu ca do res y no es sensato pensar que puedan abandonarlos. Pero, sin negar esta evidencia, pensamos que resulta deseable impulsar la autonomía y la transferencia de responsabilidades respecto de las tareas de aprendizaje.

Si el aprendizaje es la función básica de la escuela, la relación y la convivencia son también aspectos esenciales de la institución escolar. La escuela es un espacio donde viven adultos y jóvenes durante muchas horas al día. Este hecho supone una fuente de experiencias de socialización y de educación moral de gran alcance. Ninguna escuela puede olvidar la organización de la relación interpersonal y la convivencia. Nuestra intención es señalar que esta tarea recae en parte sobre los 
adultos que guían la vida escolar, pero también debe ser responsabilidad de los más jóvenes. Es necesa rio queloschi cosy chi casten gan ex periencias reales de convivencia y que puedan respon sabilizarsedecondu cirlas por sí mismos. En la dosis y la intensidad que cada caso permita, la escuela democrática debe abrir un espacio a la regulación de la vida en común.

La escuela es una institución de trabajo, de convivencia y de vida. Por tanto, en la medida que es un espacio de vida recae sobre ella la necesidad de organizar los acontecimientos que forman parte de la vida de las personas y las colectividades. En este sentido, no sabemos imaginar una escuela que no preste atención a las celebraciones propias de la comunidad, que no acoja los momentos de fiesta de sus alumnos, que no se implique en campañas reivindicativas y solidarias, 0 que no organice actividades de juego y deporte. Una escuela democrática es una escuela permeable a la sensibilidad ciudadana y a las necesidades de sus usuarios. Pero igual a como ocurría en el trabajo y en la convivencia, la animación debe ser también una oportunidad para participar.

Funciones de las as ambleas

La asamblea, en tanto que institución de diálogo, suele tener las funciones que a continuación referimos. En primer lugar, la asamblea cumple un claro papel informativo, tanto los educadores de manera vertical, como los alumnos de manera horizontal, la pueden utilizar para dar a conocer todo aquello que consideren relevante, todo aquello que atañe a la vida de la colectividad.

La asamblea es también la reunión de análisis de lo ocurrido, es el tiempo que se dedica a desentrañar el sentido de lo vivido, las causas de los problemas, o las dificultades que perturban las tareas escolares. Se trata de consi derartan to lospequeñoso gran desconflic tos de convivencia que apa recen en to daslasau las, como a cierta edad hablar del sentido de la escuela y de lo que cada alumno puede obtener de ella. Hablar incrementa la comprensión mutua entre los alumnos, la comprensión entre alumnos y profesores, y la comprensión del sentido que tiene o puede tener la escuela para cada uno de ellos.

En tercer lugar, en la asamblea, por una parte, se decide y se organiza lo que se quiere hacer $y$, por otra parte, se regula la vida de la clase. Proyectos de trabajo y pautas de convivencia son los dos resultados de esta función. No todo lo que se va a hacer pueden pensarlo los alumnos. Hay infinidad de cuestiones que están programa dasporel claustro deprofesores. Sin embargo, las asambleas pueden ser útiles para explicar el sentido de al gunastareasescolaresya previstas, paramo du larci ertos aspectos del trabajo escolar y, en algunos casos, para contribuir eficazmente a definir el tipo de trabajo a llevar a cabo.

Desde otra perspectiva, las asambleas de clase contribuyen a regular la convivencia. Por una parte, analizando los conflictos que surgen y buscando medios para apaciguarlos o bien solucionarlos. Pero también estableciendo de común acuerdo pautas y normas de conducta que vayan dibujan do un cli ma escolar positivo y sobre to do un clima escolar en el que todos, alumnos y profesores, estén implicados y se sientan corresponsables.

Con frecuencia las asambleas sirven también como encuentro catártico, como lugar para desahogarse, para decirlo todo y de hecho volver a comenzar más tranquilos. En algunos casos, más que una catarsis se trata de un comentario sobre cuestiones que son relevantes para el alumnado. Cuestiones sobre las que no se debe acordarnadanidecidir nada, pero que es conveniente que se hablen del modo más abierto posible. Hablar conjuntamente incrementa la comprensión de sí mismo y del entorno en que cada uno se mueve.

Finalmente, las asambleas son sencillamente una posibilidad abierta para que todos puedan aportar lo que deseen. 
La palabra como experiencia y compromiso

Al comenzar este apartado decíamos que es posible hacer cosas con palabras: que las asambleas no son palabrería sino experiencia y compromiso. Las asambleas son experiencia en el sentido que reclamaba Piaget (1968) cuando comparaba la adquisición de la moralidad con el aprendizaje de las distintas materias escolares. Allí afirmaba que del mismo modo que para aprender físi ca o gra máti ca no hay otro méto do que descubrir por sí mismo las leyes de la materia o del lenguaje a base de experimentosy análisis de textos, para adquirir los principios de la convivencia no hay otro sistema que colocar a los jóvenes en una situación en la que tengan que vivir de modo directo lo que significa la convivencia. De este modo adquirirán de manera activa y experiencial lo que realmente significa la vida en común, así como todas las habilidades y valores que han de ponerse en juego para lograr que sea lo más óptima posible. En las asambleaslapalabraesexperiencia.

Pero la palabra es una experiencia que orienta a los hablantes hacia el mutuo entendimiento. Es decir, mediante la palabra los interlocutores incrementan su comprensión mutua; logran elaborar planes de acción, normas de convivencia y proyectos de trabajo; y, por último, mediante la palabra se comprometen a ser coherentes con lo que han afirmado o propuesto. Participar en un proceso de comprensión y construcción conjunta a través del lenguaje compromete a todos los interlocutores. Quien participa se siente ligado y motivado a llevar a cabo aquello que se ha acordado. En las asambleasla palabra escom pro mi so (Austin, 1981).

Las asambleas son eficaces porque estamos ante una experiencia de relaciónso cial que tiende a crear en los hablantes motivación y compromiso. Sin embargo, aunque el lenguaje motiva y compromete, a menudo no culmina todavía aquello que los interlocutores han acordado. Las palabras permiten hacer cosas, pero no todas las cosas se pueden hacer con palabras. Las asambleas deben buscar la máxima coherencia entre la palabra y los comportamientos.

Por otra parte, que los alumnos y alum nas aprendan a ser ciudadanos capaces de participar en su entorno social de acuerdo a valores y criterios morales supone alcanzar distintos objetivos. Primero, que adquieran un vivo reconocimiento del valor de la colectividad y que se sientan parte del grupo donde están insertos. Que se sientan parte y que estén dispuestos a colaborar y a trabajar por el correcto funcionamiento de la comunidad. Segundo, que acepten y construyan normas; es decir, que hayan adquirido un sentido autónomo de la disciplina que les capacite para reconocer la corrección de ciertas normas escolares, para mejorarlas si es necesario y para establecer aquellas normas nuevas capaces de optimizar la convivencia. Tercero, que desarrollen una fuerte autonomía de la voluntad que les impida esconderse en el grupo y que por el contrario les impulse a participar de acuerdo a sus criterios personales en la buena marcha de la clase y del centro. Cuarto, que desarrollen el conjunto de capacidades necesarias para dialogar de modo correcto y para ser capaces de mantener una actitud reflexiva respecto de sí mismos y de la comunidad a la quepertenecen. En quin to yúl ti mo lu gar, que adquieran la predisposición a comportarse de acu erdo a va lo resta lescomo el espíri tu deiniciativa, la responsabilidad, la cooperación, la solidaridad, la tolerancia y la búsqueda de acuerdos. 


\section{Referencias bibliográficas}

APPLE, M. W.; BEANE, J . A. (Comp.) Escuelas democráticas. Madrid: Morata, 1977.

ARISTOTELES. Ética Nicomaquea. In: Tratados ético-morales. Madrid: Aguilar, 1982.

AUSTIN, J . L. Cómo hacer cosas con palabras. Barcelona: Paidós, 1981.

DARDER, P.; FRANCH, J .; COLL, C.; PELACH, J . Grupoclaseyproyec to educativodecentro. Barcelona:ICE/Horsori, 1994.

DEWEY, J. Las escuelas del mañana. Madrid: Librería de los sucesores de Hernando, 1918.

DURKHEIM, E. La educación moral. Buenos Aires: Losada, 1947.

DWORKIN, R. La comunidad liberal. Santafé de Bogotá: Universidad de los Andes y Siglo del Hombre Editores, 1996.

FERNANDEZ ENGUITA, M. Poder y participación en el sistema educativo. Barcelona: Paidós, 1992.

. La profesión docente y la comunidad escolar: crónica de un desencuentro. Madrid: Morata, 1993.

FERRIERE, A. La libertad del niño en la escuela activa. Madrid: Francisco Beltrán, 1928

FREINET, C. Por una escuela del pueblo. Barcelona: Fontanella, 1972.

. La educació moral i cívica. Barcelona: Laia, 1972.

HABERMAS, J. Pensamiento postmetafísico. Madrid: Taurus, 1990.

Teoría de la acción comunicativa. Madrid: Taurus, 1987. 2v.

. Facticidad y validez. Madrid: Trotta, 1998.

. L'intégraton républicaine. Paris: Fayard, 1996.

HUME, D. Tratado de la naturaleza humana. Madrid: Tecnos, 1988.

KOHLBERG, L.; POWER, F.C.; HIGGINS, A. La educación moral según Lawrence Kohlberg. Barcelona: Gedisa, 1997.

LOBROT, M. Pedagogía institucional. Buenos Aires: Humanitas, 1976.

LODI, M. El país errado. Barcelona: Laia, 1973.

MAKARENKO, A.S. Poema pedagógico. Barcelona: Planeta, 1983.

MEAD, G.H. Espíritu, persona y sociedad. Barcelona: Paidós, 1982.

NEILL, A.S. Summerhill. México: Fondo de Cultura Económica, 1978.

PIAGET, J .; HELLER, J. La autonomía en la escuela. Buenos Aires: Losada, 1968.

POWER, F. C.; HIGGINS, A.; KOHLBERG, L. Lawrence Kolberg's approach to moral education. New York: Columbia University Press, 1989.

PUIG, J . Feina d'educar. Barcelona: Edicions 62, 1999.

; MARTIN, X.; ESCARDIBUL, S.; NOVELLA, A. Comfomentarlaparticipa cióal'escola. Barcelona:Graó, 1997.

ROGERS, C.R. Libertad y creatividad en la educación. Barcelona: Paidós, 1975. 
SANTOS GUERRA, M. A. De mo cra cia es colar o el pro ble ma de la nie ve frita. In: AAVV: Volvera pensarlaeduca ción, política, educación y sociedad. Madrid: Morata, 1995.

VYGOTSKY, L. S. El desarrollo de los procesos psicológicos superiores. Barcelona: Crítica, 1979.

WATKINS, C.; WAGNER, P. La disciplina escolar. Barcelona: Paidós, 1991.

Recebido em 27.04.01

Aprovado em 07.06 .01

J osep Ma Puig Rovira é professor titular do Departamento de Historia e Filosofia da Educação da Faculdade de Educação da Universidade de Barcelona. É também membro do GREM (Grup de Recerca en Educació Moral) daquela universidade. Tem os seguintes livros publicado no Brasil: A construção da personalidade moral (Atica), Ética e Valores: métodos para um ensino transversal (Casa do Psicólogo), e Democracia e Participação Escolar (Moderna). 